\title{
Jubilaciones IMSS por cesantía en edad avanzada y vejez, sector agropecuario, Municipio de Navojoa, Sonora.
}

\section{IMSS retirements due to unemployment in old age and old age, agricultural sector, Municipality of Navojoa, Sonora}

\author{
Margarita Rivas Vega', María Teresa González Barajas², \\ Patricia Guadalupe Clark Coronado ${ }^{3}$
}

\author{
${ }^{1}$ https://orcid.org/0000-0002-8411-1562. Universidad Estatal de Sonora Unidad \\ Académica Navojoa. \\ ${ }^{2}$ https://orcid.org/0000-0001-7197-4337. Universidad Estatal de Sonora Unidad Académica \\ Navojoa. \\ ${ }^{3}$ https://orcid.org/0000-0001-7700-020X. Universidad Estatal de Sonora Unidad \\ Académica Navojoa. \\ DOI https://doi.org/10.46589/rdiasf.vi36.399 \\ Recibido 2 de junio 2021. \\ Aceptado 20 de septiembre 2021 \\ Publicado 24 de octubre 2021
}

\section{Resumen}

El objetivo del presente estudio fue identificar el conocimiento que existe sobre las modalidades de jubilación por cesantía en edad avanzada y vejez en el sector agropecuario, a través de una encuesta aplicada en la localidad de Masiaca en Navojoa, Sonora. El estudio es cuantitativo transversal, ya que se analizó la información en un momento determinado en el tiempo, en este caso a la fecha del levantamiento de la información; es no experimental, ya que no se manipulan las variables de interés; y tiene un alcance relacional comparativo, ya que además del diagnóstico descriptivo se realiza una comparación del nivel conocimiento de las modalidades de jubilación entre grupos distintos grupos de interés: tipo de actividad, género, grupo de edad, estratos de nivel educativo, condición de habla de lengua indígena, tipo de trabajador y condición de alta como trabajador actualmente en el IMSS.

Palabras clave: Modalidades de jubilación, cesantía en edad avanzada y vejez. 


\section{Abstract}

The objective of this study was to identify the knowledge that exists about the modalities of retirement due to unemployment in advanced age and old age in the agricultural sector, through a survey applied in the town of Masiaca in Navojoa, Sonora. The study is quantitative, cross-sectional, since the information was analyzed at a specific moment in time, in this case on the date the information was collected; it is non-experimental, since the variables of interest are not manipulated; and it has a comparative relational scope, since in addition to the descriptive diagnosis, a comparison is made of the level of knowledge of the retirement modalities between different groups of interest: type of activity, gender, age group, educational level strata, speech condition of indigenous language, type of worker and condition of registration as a worker currently in the IMSS.

Keywords: Retirement modalities, unemployment in old age and old age.

\section{Introducción}

A lo largo de la vida el ser humano se da cuenta lo necesario e importante que es tener un ahorro que los respalde a la hora de envejecer. A lo largo del ciclo de la vida el ser humano pasa por una serie de sucesos evolutivos de distinta índole. La jubilación no ha existido siempre, sino que surge como producto de las diferentes revoluciones industriales y tecnológicas que enfocan a la sociedad cada vez más hacia la producción y el consumo. Como afirma Lerh (1988), antiguamente la jubilación no existía, y al ser las familias los núcleos fundamentales de producción, el anciano no era apartado del trabajo, sino que simplemente adoptaba nuevas tareas y nuevos roles. Con ello permanecía activo y útil, representaba un valor para la familia, que a su vez se mostraba dispuesto a satisfacer las necesidades del anciano, a atenderle y cuidarle.

El desarrollo social producido por la industrialización dio lugar (Tartler, 1961 en Saenz y Meléndez, 1990), a que la persona de edad avanzada que no ejerce ya función alguna en la familia, aspire a separarse de la comunidad familiar. Esta evolución social implica que la seguridad material del anciano a cargo de la familia, ya no puede considerarse como algo natural y lógico. Así la disolución de la familia como comunidad de producción requiere un seguro de vejez independiente de la familia. Puede afirmarse que actualmente el único sector 
donde se conservan restos de este sistema es en el agrario, donde el trabajador, a pesar de recibir su jubilación, suele mantener por norma general algún grado de actividad.

Si se realiza una aproximación histórica del término jubilación se encontrará que, entre los judíos, la jubilación era un acontecimiento que se producía al terminar el período de siete semanas de años, suponía un motivo de gozo, una liberación de cargas, una ruptura con cadenas y obligaciones que habían aprisionado al individuo a lo largo de la vida. Los esclavos eran puestos en libertad, no se trabajaba durante aquel año. Esta liberación sería asimilada entre los primeros cristianos con un sentido espiritualista de perdón y liberación de culpa: es lo que en contextos religiosos llaman ganar el jubileo. A su vez los romanos adoptarían el término judío «Yobel» latinizándolo bajo el fonema judío y pasaría a las lenguas neolatinas con el significado de júbilo, pero también de jubilación (Sáez y Meléndezm 1990).

En la actualidad se puede definir la jubilación como «el momento en que de una manera u otra el anciano deja de ser productivo en bienes materiales y pasa a un segundo plano en el sistema de producción. Según González (1988), la jubilación sería «el mecanismo mediante el cual se aparta al sujeto de su trabajo al llegar a una determinada edad. $\mathrm{Su}$ fundamento social estriba en que el anciano es una persona que ya no produce, por lo que se le aparta del mundo laboral, aunque, eso sí, se le asegura mínimamente su existencia durante el resto de sus días».

Actualmente existen 2 leyes de jubilación, la ley de 1977 y la ley de 1997; el propósito de este trabajo es proporcionar información relevante sobre el tema, en especial a las personas de las comunidades, cuya principal fuente de ingresos es el sector primario, y pocas veces conocen los mecanismos para acceder a su jubilación; de ahí el interés de contribuir con información sobre el tema. Para el desarrollo de este trabajo, se realizó investigación documental sobre investigaciones del tema, encontrándose información relevante que sirvió como base para este trabajo, como el artículo de Mendizábal (1993) sobre el tema "la preparación para la jubilación, nueva ocupación del tiempo" entre otros que se citan en este documento.

En cuanto al conocimiento de las modalidades de jubilación en América Latina, diversos autores señalan que este es muy bajo. Por ejemplo, en el caso específico de Chile, 
Miranda (2013) señala que se estima que el porcentaje de desconocimiento de las modalidades de jubilación es de 90\%. En el caso de México, de acuerdo con Ruiz-Medina et al (2016), el porcentaje de conocimiento de las modalidades de pensión por invalidez y cesantía o vejez es de 10 y $17 \%$, respectivamente.

Por otra parte, la revisión de la literatura permite constatar que no hay muchas investigaciones con relación a este tema.

El problema de investigación que guía los esfuerzos de este trabajo, es la importancia de que toda persona con una vida laboral activa, al término de dicha etapa, conozca las modalidades existentes con las cuales puede obtener una jubilación digna para vivir, dado que existen personas que se dedican al sector primario ya sea agricultura o ganadería, y no cuentan con seguro médico ya que no están cotizando con patrón y desconocen las diferentes modalidades a las que pueden tener acceso, y quedan totalmente desprotegidos al momento de alguna enfermedad o incluso al momento de su vejez, y tienen que trabajar ya estando en una edad muy avanzada, lo cual representa un problema social.

La población económicamente activa del municipio en estudio es de 46,786 habitantes; mientras que la económicamente inactiva asciende a 56,559 habitantes. Al sector primario le conciernen: 7,923, le corresponde un porcentaje del 17.2\% del total de la población activa en el sector primario, de las cuales cierto porcentaje no cuentan con una afiliación al seguro social, por lo que no cuentan con servicio médico y mucho menos a una jubilación digna que pueda respaldar su futuro económico.

El objetivo general de este trabajo es determinar y caracterizar el grado de conocimiento que tienen los trabajadores del campo (agrícolas y ganaderos), sobre las modalidades de jubilación del IMSS, considerando para ello a la Localidad de Masiaca del municipio de Navojoa, del estado de Sonora, México. Los objetivos específicos fueron:

1. Llevar a cabo un estudio descriptivo, a fin de caracterizar a los encuestados.

2. Previa validación del instrumento, construir un índice de conocimiento de las modalidades de jubilación (ICMJ) del IMSS.

3. Estimar la proporción de trabajadores del campo cuyo conocimiento es mayor o igual a los 50 puntos del índice previamente construido. 
4. Efectuar un estudio comparativo por género, grupo de edad, estratos de nivel educativo, condición de habla de lengua indígena, tipo de trabajador y condición de alta como trabajador actualmente en el IMSS, para determinar si existen o no diferencias significativas en el nivel de conocimiento que sobre las modalidades de jubilación tienen los trabajadores agrícolas y ganaderos objeto de estudio.

Los principales resultados se presentan en el apartado correspondiente.

\section{Método}

El estudio es cuantitativo transversal, ya que se analiza la información en un momento determinado en el tiempo, en este caso a la fecha del levantamiento de la información; es no experimental, ya que no se manipulan las variables de interés; y tiene un alcance relacional comparativo, ya que además del diagnóstico descriptivo se realiza una comparación del nivel conocimiento de las modalidades de jubilación entre grupos distintos grupos de interés: tipo de actividad, género, grupo de edad, estratos de nivel educativo, condición de habla de lengua indígena, tipo de trabajador y condición de alta como trabajador actualmente en el IMSS. Hipótesis

H1: Se presentan diferencias significativas en el nivel de conocimiento que tienen los trabajadores agrícolas y ganaderos con respecto a las modalidades de jubilación del IMSS.

$\mathrm{H} 2$ : Se presentan diferencias significativas por género en el nivel de conocimiento que tienen los trabajadores del campo, con respecto a las modalidades de jubilación del IMSS.

H3: Se presentan diferencias significativas por estratos de niveles de estudio, en el grado de conocimiento que tienen los trabajadores del campo, con respecto a las modalidades de jubilación del IMSS.

H4: Se presentan diferencias significativas por condición de habla de lengua indígena en el nivel de conocimiento que tienen los trabajadores del campo, con respecto a las modalidades de jubilación del IMSS.

H5: Se presentan diferencias significativas por tipo de trabajador (asalariado o no asalariado) en el nivel de conocimiento que tienen los trabajadores del campo, con respecto a las modalidades de jubilación del IMSS. 
H6: Se presentan diferencias significativas por condición de alta como trabajador actualmente en el IMSS en el nivel de conocimiento que tienen los trabajadores del campo, con respecto a las modalidades de jubilación del IMSS.

Selección de la muestra

El tamaño de muestra se obtuvo para la proporción de trabajadores del campo con nivel de conocimiento mayor a 50 puntos del índice que se construye para tal efecto, considerando una confianza de $95 \%$ y un error de estimación de $10 \%$ y que, de acuerdo con diversos autores como Anderson, Sweenwy, \& Williams (2012), se obtiene mediante la siguiente expresión:

$$
n=\frac{Z^{2} \pi(1-\pi)}{E^{2}}
$$

Donde:

$Z \quad$ Es el valor de este estadístico que corresponde a la confianza considerada del 95\%. En este caso, $Z=1.96$

$\pi \quad$ Es la proporción esperada de productores que se considera destinan su producción al consumidor, en este caso se considero $\pi=0.5$, es decir, $50 \%$, ya que dicha proporción genera el mayor tamaño de muestra para una confianza y error de estimación determinados.

$E$ Es el error de estimación considerado, en este caso $E=0.10$, es decir, $10 \%$.

Con los valores considerados, la muestra resulta ser de 97 trabajadores del campo, considerando finalmente un tamaño de muestra efectivo de 120 trabajadores.

Recolección de datos

Selección del Instrumento

Validez

a) Validez de contenido

El cuestionario de la encuesta fue revisado en cuanto a la pertinencia de su contenido, por profesores e investigadores de la Unidad Académica Navojoa de la Universidad Estatal de Sonora y de la Facultad de Contaduría y Administración de la Universidad Autónoma de Yucatán (UADY).

b) Validez de constructo y confiabilidad 
La validación del instrumento propuesto se llevó a cabo mediante la aplicación del análisis factorial exploratorio y confirmatorio, a fin de verificar la validez de constructo y mediante la obtención del coeficiente alfa de Cronbach, para revisar su confiabilidad.

El constructo considerado en este caso, es el conocimiento que los trabajadores del campo tienen sobre las modalidades de jubilación del IMSS. La información se obtuvo a partir las preguntas formuladas mediante tres reactivos en escala Likert. Los resultados se describen a continuación.

El coeficiente de adecuación muestral de Kaiser Meyer y Olkin (KMO) fue de 0.754, el cual se considera satisfactorio, registrando la prueba de Bartlett un valor $\mathrm{p}=0.000$, lo que indica que se considera que la relación entre las variables que conforman el constructo es adecuada, siendo la variabilidad explicada por el primer componente de $93.4 \%$, lo que permite construir a partir de dichos reactivos un índice representativo.

En cuanto al confiabilidad, el coeficiente alfa de Cronbach registró el valor 0.959 el cual se considera satisfactorio.

De acuerdo con los resultados anteriores el instrumento se considera que tiene validez y confiabilidad.

Los resultados fueron obtenidos mediante el empleo del paquete estadístico IBM SPSS versión 21.

\section{Resultados}

Estudio descriptivo

De las 120 personas encuestadas del municipio de la Localidad de Masiaca del municipio de Navojoa, la edad promedio del grupo encuestado es de 57 años, con una edad mínima de 26 y una edad máxima de 89 años. De este grupo, 24 personas (20\%) son mujeres y 96 personas $(80 \%)$ son hombres.

Realizando una clasificación por grupos quinquenales de edad y género, se obtuvo la tabla 1 que específica en números de caso de cada quinquenio por género, observando que el mayor número de personas encuestadas mujeres se encuentra en el rango de 36 a 40 años y el de hombres entre 61 y 65 años. 
Tabla 1. Personas encuestadas según genero y rango de edad

\begin{tabular}{|c|c|c|c|c|c|c|}
\hline $\begin{array}{c}\text { Rango de } \\
\text { Edad }\end{array}$ & Mujer & Porcentaje & Hombre & Porcentaje & Total & Porcentaje \\
\hline $26-30$ & 0 & $0.0 \%$ & 3 & $3.1 \%$ & 3 & $2.5 \%$ \\
\hline $31-35$ & 2 & $8.3 \%$ & 5 & $5.2 \%$ & 7 & $5.8 \%$ \\
\hline $36-40$ & 5 & $20.8 \%$ & 4 & $4.2 \%$ & 9 & $7.5 \%$ \\
\hline $41-45$ & 1 & $4.2 \%$ & 11 & $11.5 \%$ & 12 & $10.0 \%$ \\
\hline $46-50$ & 2 & $8.3 \%$ & 10 & $10.4 \%$ & 12 & $10.0 \%$ \\
\hline $51-55$ & 1 & $4.2 \%$ & 7 & $7.3 \%$ & 8 & $6.7 \%$ \\
\hline $56-60$ & 2 & $8.3 \%$ & 12 & $12.5 \%$ & 14 & $11.7 \%$ \\
\hline $61-65$ & 2 & $8.3 \%$ & 16 & $16.7 \%$ & 18 & $15.0 \%$ \\
\hline $66-70$ & 3 & $12.5 \%$ & 10 & $10.4 \%$ & 13 & $10.8 \%$ \\
\hline $71-75$ & 2 & $8.3 \%$ & 8 & $8.3 \%$ & 10 & $8.3 \%$ \\
\hline $76-80$ & 2 & $8.3 \%$ & 2 & $2.1 \%$ & 4 & $3.3 \%$ \\
\hline $81-85$ & 1 & $4.2 \%$ & 5 & $5.2 \%$ & 6 & $5.0 \%$ \\
\hline $86-90$ & 1 & $4.2 \%$ & 3 & $3.1 \%$ & 4 & $3.3 \%$ \\
\hline Total & 24 & $100.0 \%$ & 96 & $100.0 \%$ & 120 & $100.0 \%$ \\
\hline
\end{tabular}

Fuente: Elaboración propia a partir de los datos obtenidos

A continuación, se presenta la gráfica correspondiente al número de casos por quinquenio de edad y género.

\section{Gráfica 1. Personas encuestadas según género y rango de edad}

\section{Número de casos encuestados por rango de edad y género}

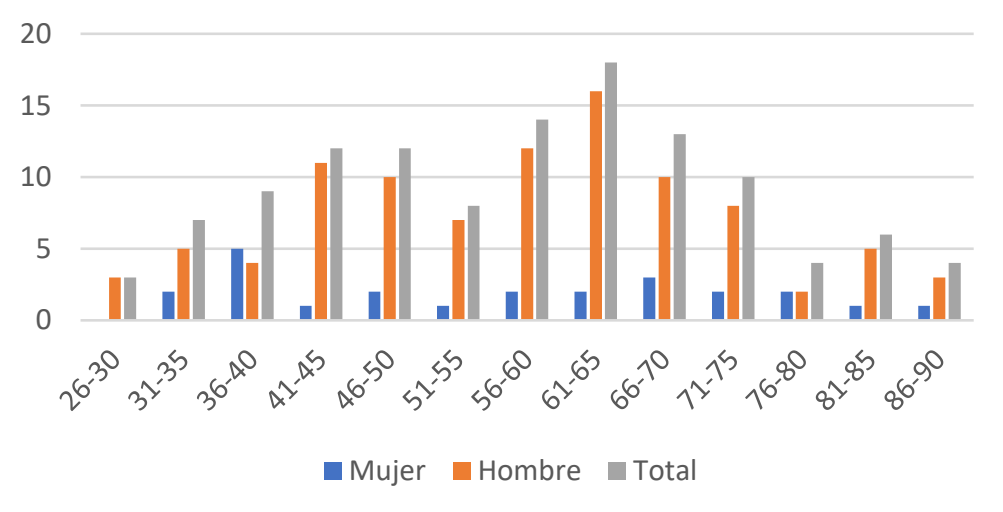

Fuente: Elaboración propia a partir de los datos obtenidos

De los datos obtenidos sobre los estudios concluidos, el 26\% tiene primaria concluida, el $32.5 \%$ tiene secundaria concluida y el $8.3 \%$ tiene bachillerato concluido, según se muestra el Tabla 2. 
Tabla 2. Nivel de estudios

\begin{tabular}{|l|l|l|}
\hline Nivel de Estudios & Frecuencia & Porcentaje \\
\hline Sin Instrucción & 3 & $2.5 \%$ \\
\hline Primaria Incompleta & 27 & $22.5 \%$ \\
\hline Primaria & 32 & $26.7 \%$ \\
\hline Secundaria & 39 & $32.5 \%$ \\
\hline Bachillerato & 10 & $8.3 \%$ \\
\hline Licenciatura & 8 & $6.7 \%$ \\
\hline Postgrado & 1 & $0.8 \%$ \\
\hline Total & 120 & $100.0 \%$ \\
\hline
\end{tabular}

Fuente: Elaboración propia a partir de los datos obtenidos

El comportamiento del nivel de estudios por género puede observarse en la gráfica 2, la cual muestra que el nivel de estudios con mayor porcentaje es secundaria concluida, $33.3 \%$ mujeres y $32.3 \%$ hombres.

\section{Gráfica 2. Nivel de estudios}

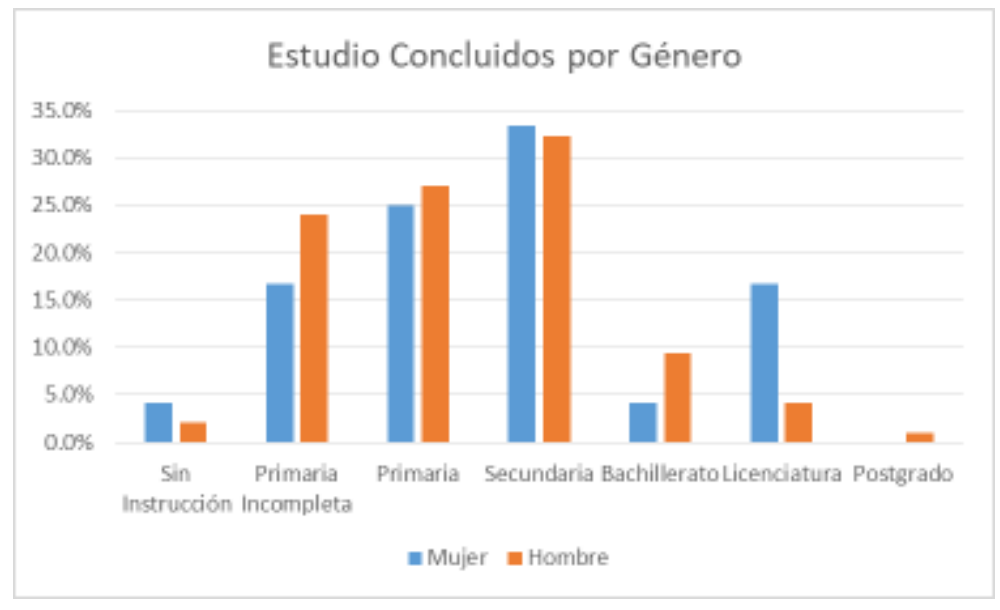

Fuente: Elaboración propia a partir de los datos obtenidos

Uno de los resultados muestra que el $40.8 \%$ de la población habla lengua indígena, según los resultados mostrados en la Tabla 3. 
Tabla 3. Lengua indígena

\begin{tabular}{|c|c|c|}
\hline Habla Lengua Indígena & Frecuencia & Porcentaje \\
\hline $\mathrm{Si}$ & 49 & $40.8 \%$ \\
\hline No & 71 & $59.2 \%$ \\
\hline Total & 120 & $100.0 \%$ \\
\hline
\end{tabular}

Fuente: Elaboración propia a partir de los datos obtenidos

De los datos recolectados, podemos observar que de las dos actividades principales el $25.8 \%$ se dedican a la agricultura, mientas el $74.2 \%$ se dedican a la ganadería, como puede observarse en la Tabla 4.

Tabla 4. Actividad principal

\begin{tabular}{|l|c|c|}
\hline $\begin{array}{c}\text { Actividad } \\
\text { Principal }\end{array}$ & Frecuencia & Porcentaje \\
\hline Agricultura & 31 & $25.8 \%$ \\
\hline Ganadería & 89 & $74.2 \%$ \\
\hline Total & 120 & $100.0 \%$ \\
\hline
\end{tabular}

Fuente: Elaboración propia a partir de los datos obtenidos

Dentro de la actividad de la agricultura, como muestra la Tabla 5, se consideraron cuatro de los principales cultivos producidos en la región, maíz, frijol, trigo y sorgo, resultando que de los encuestados el cultivo de mayor producción es el frijol con un 32.3\% y dentro del cultivo clasificado como "otro" es el garbanzo que representa el $48.5 \%$ de la producción.

Tabla 5. Cultivo principal

\begin{tabular}{|l|l|l|l|}
\hline $\begin{array}{l}\text { Cultivo } \\
\text { Principal }\end{array}$ & Frecuencia & Porcentaje & $\begin{array}{l}\text { Porcentaje } \\
\text { acumulado }\end{array}$ \\
\hline Maíz & 3 & $9.7 \%$ & $9.7 \%$ \\
\hline Frijol & 10 & $32.3 \%$ & $41.9 \%$ \\
\hline Trigo & 1 & $3.2 \%$ & $45.2 \%$ \\
\hline Sorgo & 2 & $6.5 \%$ & $51.6 \%$ \\
\hline Otro & 15 & $48.4 \%$ & $100.0 \%$ \\
\hline Total & 31 & $100.0 \%$ & \\
\hline
\end{tabular}

Fuente: Elaboración propia a partir de los datos obtenidos 
A continuación, se presenta la gráfica 3 por tipo de cultivo.

\section{Gráfica 3. Cultivo principal}

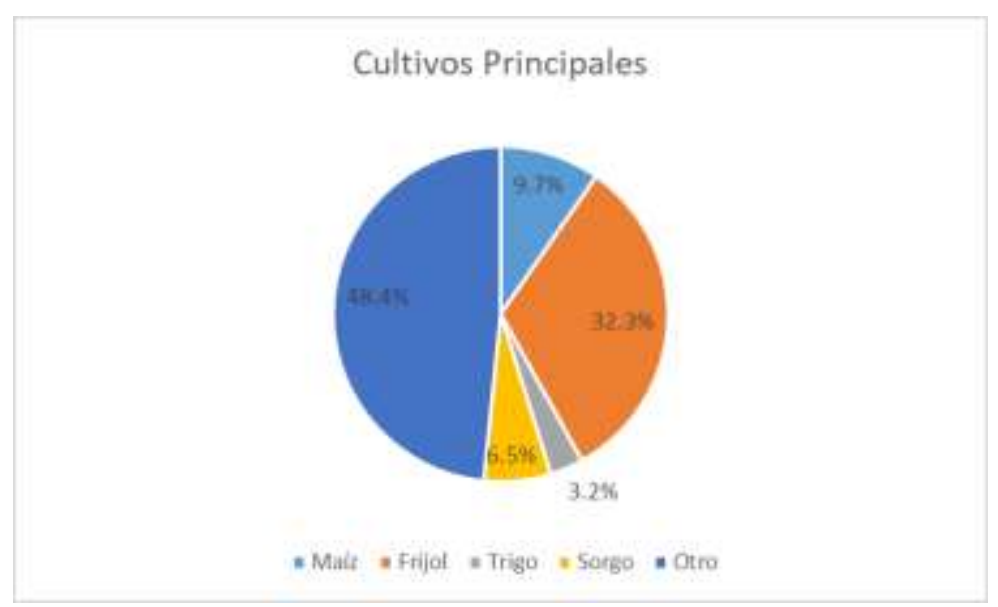

Fuente: Elaboración propia a partir de los datos obtenidos

En el caso de la actividad de la ganadería, como se muestra en la Tabla 6, se consideraron cinco especies ganaderas producidas en la zona, ganado bovino, ganado porcino, ganado ovino, ganado caprino y aves. Siendo el ganado bovino el de mayor producción con el 75\% de los casos y en segundo lugar el ganado caprino con el $15 \%$ del total.

\section{Tabla 6. Especies ganaderas}

\begin{tabular}{|l|c|c|c|}
\hline $\begin{array}{c}\text { Especies } \\
\text { Ganaderas }\end{array}$ & Frecuencia & Porcentaje & $\begin{array}{c}\text { Porcentaje } \\
\text { acumulado }\end{array}$ \\
\hline Bovino & 67 & $75.3 \%$ & $75.3 \%$ \\
\hline Porcino & 3 & $3.4 \%$ & $78.7 \%$ \\
\hline Ovino & 3 & $3.4 \%$ & $82.0 \%$ \\
\hline Caprino & 14 & $15.7 \%$ & $97.8 \%$ \\
\hline Aves & 1 & $1.1 \%$ & $98.9 \%$ \\
\hline Otra & 1 & $1.1 \%$ & $100.0 \%$ \\
\hline Total & 89 & $100.0 \%$ & \\
\hline
\end{tabular}

Fuente: Elaboración propia a partir de los datos obtenidos

La mención a la especie que se clasificó como otra es la de ganado equino, como se observa en la gráfica 4. 


\section{Gráfica 4. Especies ganaderas}

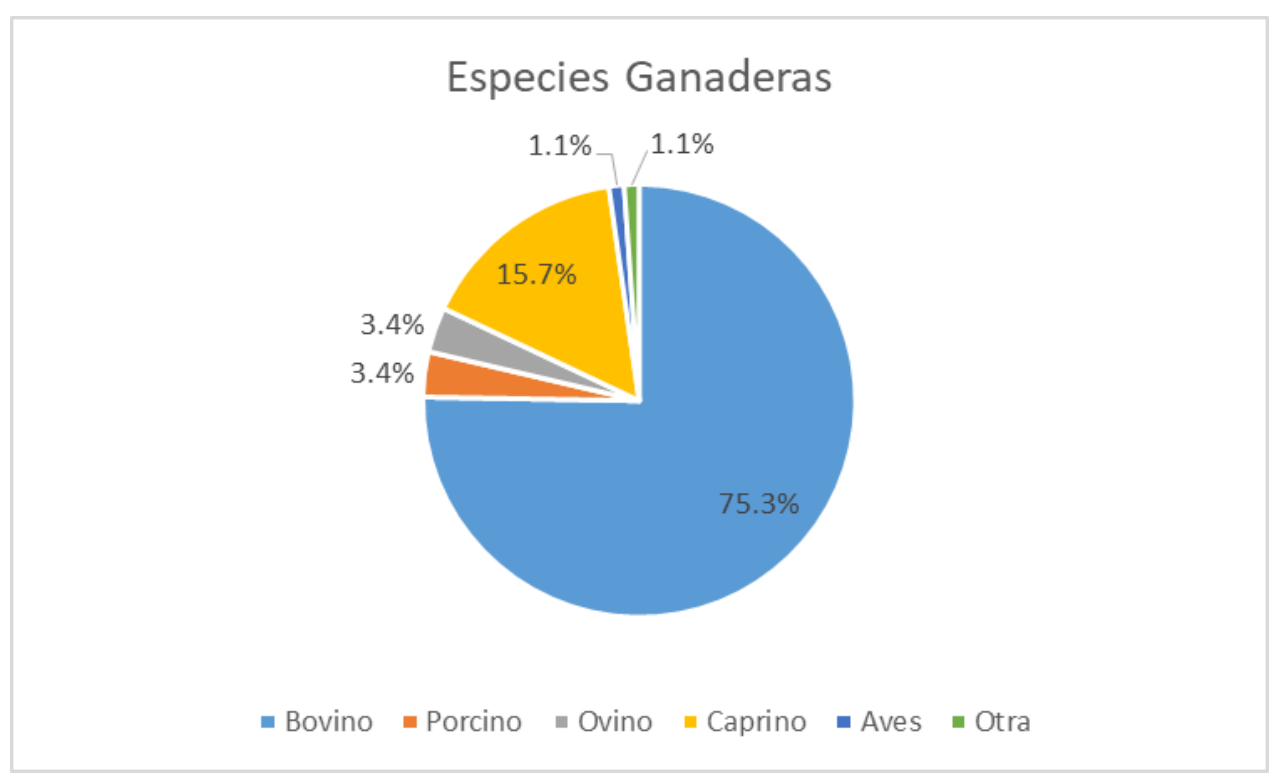

Fuente: Elaboración propia a partir de los datos obtenidos

Dentro de la encuesta se plantea la pregunta de si ¿Es derechohabiente actualmente?, a la cual el $71.1 \%$ responde de forma afirmativa y tan solo el $28.3 \%$ responde en forma negativa, los resultados se muestran en la Tabla 7.

Tabla 7. Derechohabientes actuales

\begin{tabular}{|c|c|c|}
\hline $\begin{array}{c}\text { Es derechohabiente } \\
\text { actualmente? }\end{array}$ & Frecuencia & Porcentaje \\
\hline $\mathrm{Si}$ & 86 & 71.7 \\
\hline No & 34 & 28.3 \\
\hline Total & 120 & 100.0 \\
\hline
\end{tabular}

Fuente: Elaboración propia a partir de los datos obtenidos

De las 86 personas que actualmente son derechohabientes en alguna institución, en la Tabla 8 se muestra en cuál de las instituciones ha sido dado de alta como derechohabiente, mostrando que 38 casos que representan el $44.2 \%$ están actualmente registrados en el INSABI, el $36.0 \%$ en el IMSS y el $17.4 \%$ en el ISSSTE. 
Tabla 8. Derechohabientes por institución de seguridad social

\begin{tabular}{|l|c|c|c|}
\hline \multicolumn{1}{|c|}{ INSTITUCIÓN } & Frecuencia & Porcentaje & $\begin{array}{c}\text { Porcentaje } \\
\text { acumulado }\end{array}$ \\
\hline IMSS & 31 & $36.0 \%$ & $36.0 \%$ \\
\hline ISSSTE & 15 & $17.4 \%$ & $53.5 \%$ \\
\hline INSABI (Seguro Popular) & 38 & $44.2 \%$ & $97.7 \%$ \\
\hline Otra & 2 & $2.3 \%$ & $100.0 \%$ \\
\hline Total & 86 & $100.0 \%$ & \\
\hline
\end{tabular}

Fuente: Elaboración propia a partir de los datos obtenidos

De las 120 personas encuestadas, actualmente se encuentran dados de alta en el IMSS el $18.3 \%$, como muestra la Tabla 9.

\section{Tabla 9. Derechohabientes en el IMSS}

\begin{tabular}{|c|r|r|}
\hline $\begin{array}{c}\text { Acualmenete } \\
\text { está dado de } \\
\text { alta en el IMSS }\end{array}$ & Frecuencia & Porcentaje \\
\hline $\mathrm{Si}$ & 22 & $18.3 \%$ \\
\hline $\mathrm{No}$ & 98 & $81.7 \%$ \\
\hline Total & 120 & $100.0 \%$ \\
\hline
\end{tabular}

Fuente: Elaboración propia a partir de los datos obtenidos

La Tabla 10 muestra que el número de personas que han estado registrados como derechohabiente en el algún momento de su vida, en el pasado resultaron 56 que representa el $46.7 \%$.

Tabla 10. Derechohabientes que estuvieron dados de alta en el pasado

\begin{tabular}{|c|c|c|}
\hline $\begin{array}{c}\text { En el pasado tuvo alta } \\
\text { como trabajador en el } \\
\text { IMSS }\end{array}$ & Frecuencia & Porcentaje \\
\hline $\mathrm{Si}$ & 56 & $46.7 \%$ \\
\hline $\mathrm{No}$ & 64 & $53.3 \%$ \\
\hline Total & 120 & $100.0 \%$ \\
\hline
\end{tabular}

Fuente: Elaboración propia a partir de los datos obtenidos

Como sabemos existen diferentes formas de hacer frente a la situación de llegar a la vejez y sin poder generar ingresos propios, ya que no todas las personas cuentan con un plan 
de pensiones institucional, en la encuesta se pregunta sobre si cuenta con un plan para su vejez, resultando que el $41.7 \%$ considera la opción de seguir trabajando, y tan solo el $10 \%$ cuenta con una pensión del IMSS. La Tabla 11 muestra las opciones consideradas.

Tabla 11. Plan para la vejez

\begin{tabular}{|c|c|c|}
\hline Plan para su vejez & Frecuencia & Porcentaje \\
\hline Algún familiar se hará cargo & 24 & $20.0 \%$ \\
\hline Con ayuda del gobierno & 24 & $20.0 \%$ \\
\hline Seguirá trabajando & 50 & $41.7 \%$ \\
\hline Pensión del IMSS & 10 & $8.3 \%$ \\
\hline Otra & 12 & $10.0 \%$ \\
\hline Total & 120 & $100.0 \%$ \\
\hline
\end{tabular}

Fuente: Elaboración propia a partir de los datos obtenidos

Las Tablas 13, 14 y 15 muestran las tres opciones de las modalidades de jubilación del IMSS, Modalidad 13, Modalidad 40 y Modalidad 43. Dichas tablas presentan los cinco niveles de conocimiento que se consideraron en el estudio, a saber, nada, poco, más o menos, bien, y muy bien.

La Tabla 13 se muestra el nivel de conocimiento de la Modalidad 13, en la que puede observarse que de los encuestados, el $65.8 \%$ no la conoce, el $20.8 \%$ la conoce poco, lo que indica el $86.7 \%$ de las personas conocen poco o nada de la Modalidad 13; el 9.2\% la conoce más o menos, el $3.3 \%$ la conoce bien y tan solo el $0.8 \%$ la conoce muy bien.

Tabla 13. Nivel de conocimiento de la Modalidad 13

\begin{tabular}{|l|r|r|r|}
\hline \multicolumn{1}{|c|}{ Modalidad 13 } & Frecuencia & Porcentaje & $\begin{array}{r}\text { Porcentaje } \\
\text { acumulado }\end{array}$ \\
\hline Nada & 79 & $65.8 \%$ & $65.8 \%$ \\
\hline Poco & 25 & $20.8 \%$ & $86.7 \%$ \\
\hline Más o menos & 11 & $9.2 \%$ & $95.8 \%$ \\
\hline Bien & 4 & $3.3 \%$ & $99.2 \%$ \\
\hline Muy bien & 1 & $0.8 \%$ & $100.0 \%$ \\
\hline Total & 120 & $100.0 \%$ & \\
\hline
\end{tabular}

Fuente: Elaboración propia a partir de los datos obtenidos 
En la Tabla 14 se muestra el nivel de conocimiento de la Modalidad 40; en ella puede observarse que de los encuestados, el $70.8 \%$ no la conoce nada, el $15.8 \%$ la conoce poco, lo que indica que el $86.6 \%$ de las personas conocen poco o nada de la Modalidad 40; el 10.8\% la conoce más o menos, el $1.7 \%$ conoce bien y tan solo el $0.8 \%$ la conoce muy bien.

Tabla 14. Nivel de conocimiento de la Modalidad 40

\begin{tabular}{|l|r|r|r|}
\hline \multicolumn{1}{|c|}{ Modalidad 40 } & Frecuencia & Porcentaje & $\begin{array}{r}\text { Porcentaje } \\
\text { acumulado }\end{array}$ \\
\hline Nada & 85 & $70.8 \%$ & $70.8 \%$ \\
\hline Poco & 19 & $15.8 \%$ & $86.7 \%$ \\
\hline Más o menos & 13 & $10.8 \%$ & $97.5 \%$ \\
\hline Bien & 2 & $1.7 \%$ & $99.2 \%$ \\
\hline Muy bien & 1 & $0.8 \%$ & $100.0 \%$ \\
\hline Total & 120 & $100.0 \%$ & \\
\hline
\end{tabular}

Fuente: Elaboración propia a partir de los datos obtenidos

En la Tabla 15 se muestra el nivel de conocimiento de la Modalidad 43, en la que puede observarse que de los encuestados, el $76.7 \%$ no la conoce, el $11.7 \%$ la conoce poco, lo que indica el $88.3 \%$ de las personas conocen poco o nada de la Modalidad 43, el 9.2\% la conoce más o menos, el $1.7 \%$ la conoce bien y tan solo el $0.8 \%$ la conoce muy bien.

Tabla 15. Nivel de conocimiento de la Modalidad 43

\begin{tabular}{|l|r|r|r|}
\hline \multicolumn{1}{|c|}{ Modalidad 43 } & Frecuencia & Porcentaje & $\begin{array}{r}\text { Porcentaje } \\
\text { acumulado }\end{array}$ \\
\hline Nada & 92 & $76.7 \%$ & $76.7 \%$ \\
\hline Poco & 14 & $11.7 \%$ & $88.3 \%$ \\
\hline Más o menos & 11 & $9.2 \%$ & $97.5 \%$ \\
\hline Bien & 2 & $1.7 \%$ & $99.2 \%$ \\
\hline Muy bien & 1 & $0.8 \%$ & $100.0 \%$ \\
\hline Total & 120 & $100.0 \%$ & \\
\hline
\end{tabular}

Fuente: Elaboración propia a partir de los datos obtenidos 
La Gráfica 5 siguiente muestra los niveles de conocimiento de las tres modalidades.

\section{Gráfica 5. Nivel de conocimiento de las modalidades de jubilación}

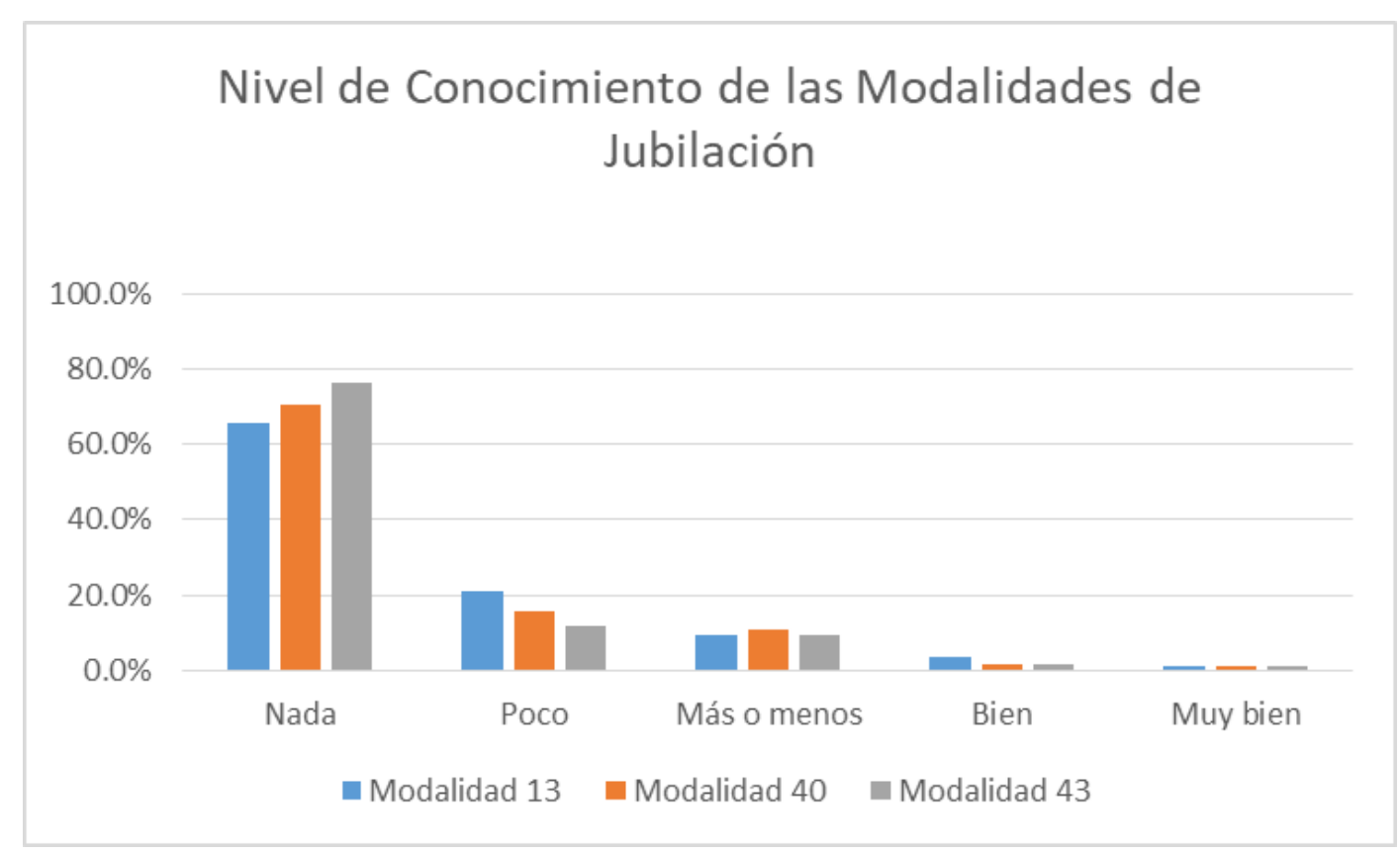

Fuente: Elaboración propia a partir de los datos obtenidos

\section{Construcción del índice de conocimiento}

A fin de llevar a cabo la estimación del nivel de conocimiento y los estudios comparativos por tipo de actividad, género, grupo de edad, estratos de nivel educativo, condición de habla de lengua indígena, tipo de trabajador y condición de alta como trabajador actualmente en el IMSS, se construyó el índice de Conocimiento de las Modalidades de Jubilación (ICMJ), a partir de las respuestas a los reactivos en escala ordinal.

Este índice se presenta en escala de 0 a 100, para cada unidad de observación (trabajador), a partir de las sumas de las respuestas a los reactivos en escala ordinal, considerando para ello la siguiente expresión:

$$
I M C J_{j}=\frac{V_{\text {max }}\left(\sum_{i=1}^{n} x_{i j}-V_{\text {minant }}\right)}{V_{\text {maxant }}-V_{\text {minant }}}
$$

donde $I C M J_{j}$ es el valor del indicador correspondiente a la unidad de observación (trabajador) j, la sumatoria corresponde al acumulado de puntos alcanzado en la escala ordinal original de acuerdo al nivel de conocimiento de cada modalidad, Vmax es el valor máximo en la nueva 
escala (es decir, 100) y Vmaxant y Vminant son los valores máximo y mínimo en la escala ordinal 1 a 5 original, en este caso, 15 y 3 respectivamente.

En la Tabla 16 y gráfica 6, se muestra una breve estadística descriptiva y el histograma asociado con los valores de este índice.

Tabla 16. Índice de conocimiento de las modalidades de jubilación

\begin{tabular}{|l|l|}
\hline $\mathrm{N} \quad$ Válidos & 120 \\
Media & 0 \\
Mediana & 11.385 \\
Desv. típ. & .000 \\
Asimetría & 18.3956 \\
Curtosis & 1.912 \\
Mínimo & 3.446 \\
Máximo & .0 \\
\hline
\end{tabular}

Fuente: Elaboración propia a partir de los datos obtenidos

Gráfica 6. Índice de conocimiento de las modalidades de jubilación (ICMJ)

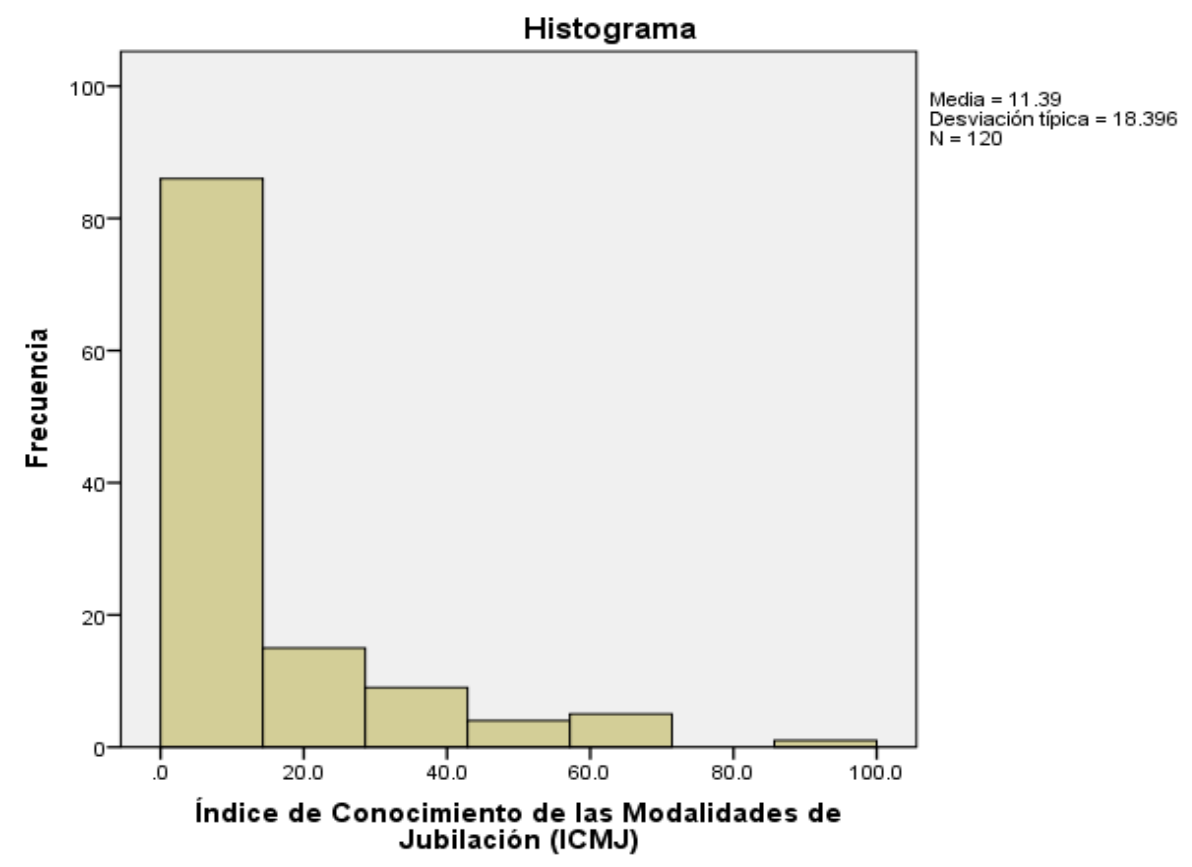

Fuente: Elaboración propia a partir de los datos obtenidos 
Estimación por Intervalos.

La expresión para la estimación del intervalo de confianza, para la proporción de trabajadores del campo que tienen un nivel de conocimiento superior o igual a los 50 puntos del índice previamente construido, se presenta a continuación.

$$
p \pm Z \sqrt{\frac{p(1-p)}{n}}
$$

Donde:

$p$ es la estimación puntual de la proporción de trabajadores del campo que tienen un nivel de conocimiento mayor o igual a los 50 puntos del índice de conocimiento de las modalidades de jubilación del IMSS (ICMJ), como resultado del muestreo; en esta caso, $\mathrm{p}=8.3 \%$ $Z$ es el valor del estadístico $Z$ para porcentaje de confianza establecido para llevar a cabo el estudio, en este caso $95 \%$, de modo que $\mathrm{Z}=1.96$

$n$ es el tamaño de muestra considerado para el estudio, en este caso, el tamaño de muestra efectivo fue de $\mathrm{n}=120$ trabajadores.

De acuerdo con lo anterior, la estimación del porcentaje de trabajadores del campo de la localidad de Masiaca que tienen un nivel de conocimiento mayor o igual a los 50 puntos del índice de conocimiento de las modalidades de jubilación del IMSS (ICMJ), es de 8.3\%, con un margen de error de $4.9 \%$ y una confianza del $95 \%$, es decir, que el valor de dicho porcentaje en la comunidad objeto de estudio, se encuentra en un intervalo que va del $3.4 \%$ a $13.3 \%$, teniendo para ello un $95 \%$ de confianza.

Resultados similares fueron obtenidos en el caso específico de los trabajadores agrícolas y de los trabajadores ganaderos. Un resumen de los resultados obtenidos se presenta en la Tabla 17. 
Tabla 17. Resultados de la estimación por intervalos (95\%)

\begin{tabular}{|l|c|c|c|c|}
\hline Ámbito & $\begin{array}{l}\text { Estimación } \\
\text { puntual }\end{array}$ & Margen de error & Límite inferior & Límite superior \\
\hline $\begin{array}{l}\text { Trabajadores } \\
\text { del campo }\end{array}$ & 8.3 & 4.9 & 3.4 & 13.3 \\
\hline Agricultores & 3.2 & 3.2 & 0.0 & 6.4 \\
\hline Ganaderos & 10.1 & 5.4 & 4.7 & 15.5 \\
\hline
\end{tabular}

Fuente: Elaboración propia a partir de los datos obtenidos

Como se observa, el porcentaje trabajadores del campo (agricultores y ganaderos), con un conocimiento de las modalidades de jubilación del IMSS, igual o superior a los 50 puntos del índice, es bajo, ya que en todos los casos es inferior al 11\%. No obstante, dicho porcentaje es mayor en el caso de los ganaderos, con respecto al valor observado en el caso de los agricultores.

Pruebas de hipótesis (criterio del valor $\mathrm{p}$ )

Para la verificación de las pruebas descritas a continuación se tomó en consideración el criterio del valor p. Dicho valor corresponde al área bajo la distribución de probabilidad del estimador del parámetro poblacional correspondiente al valor del estadístico de prueba, de modo que, si dicho valor es menor o igual que el nivel de significancia de la prueba, se rechaza la hipótesis nula y en caso contrario no se rechaza. En general, en los cuadros de resultados del presente documento, se presenta el valor p asociado con la verificación de las pruebas de hipótesis efectuadas.

Pruebas de normalidad.

A fin de establecer la prueba adecuada a aplicar para los estudios comparativos, mostrada en la Tabla 18, se llevó a cabo la prueba de normalidad de Kolomogorov Smirnov al índice construido. Esta prueba permite verificar la hipótesis nula de que la variable de interés (en nuestro caso el índice) tiene o no una determinada distribución de probabilidad, en el caso del presente estudio, la distribución normal, considerando como Ho que la variable presenta una distribución de probabilidad normal. 
Tabla 18. Prueba de Kolmogorov-Smirnov para una muestra

\begin{tabular}{|c|c|c|}
\hline & & $\begin{array}{l}\text { Índice de Conocimiento } \\
\text { de las Modalidades de } \\
\text { Jubilación (ICMJ) }\end{array}$ \\
\hline \multicolumn{2}{|l|}{$\mathrm{N}$} & 120 \\
\hline Parámetros & Media & 11.385 \\
\hline normales $^{\mathrm{a}, \mathrm{b}}$ & Desviación típica & 18.3956 \\
\hline \multirow{3}{*}{$\begin{array}{l}\text { Diferencias más } \\
\text { extremas }\end{array}$} & Absoluta & .299 \\
\hline & Positiva & .299 \\
\hline & Negativa & -.268 \\
\hline \multicolumn{2}{|c|}{$\mathrm{Z}$ de Kolmogorov-Smirnov } & 3.272 \\
\hline \multicolumn{2}{|c|}{ Sig. asintót. (bilateral) } & .000 \\
\hline
\end{tabular}

a. La distribución de contraste es la Normal.

b. Se han calculado a partir de los datos.

Fuente: Elaboración propia a partir de los datos obtenidos

Como resultado, dado que el valor $\mathrm{p}=0.000$ (aparece como sig. en la parte inferior de la tabla anterior), se determinó que el índice no presenta una distribución normal. Al no verificarse la normalidad del índice, la prueba adecuada a efectuar para comparar el índice es la prueba U de Mann Whitney.

Pruebas de hipótesis (estudio comparativo).

La prueba $\mathrm{U}$ de Mann Whitney es el equivalente no paramétrico de la prueba t de Student para dos muestras independientes y se emplea en el caso de que la variable sea ordinal o al ser cuantitativa no se cumplen los supuestos de la prueba t de Student, en particular el supuesto de normalidad. La hipótesis nula corresponde a que no existen diferencias estadísticamente significativas en los valores medios (medias o medianas, según el tipo de variable) de los grupos a comparar (una descripción más detallada sobre esta prueba se presenta en el Anexo). 
Los resultados obtenidos de las pruebas comparativas del ICMJ efectuadas por tipo de actividad, género, grupo de edad, estratos de nivel educativo, condición de habla de lengua indígena, tipo de trabajador y condición de alta como trabajador actualmente en el IMSS, se presentan en la Tabla 19.

Tabla 19. Pruebas comparativas del ICMJ

\begin{tabular}{|l|l|l|}
\hline Variables & Valor p & Resultado \\
\hline Tipo de actividad & $0.005^{* * *}$ & E.S. \\
\hline Género & 0.333 & N.S. \\
\hline Grupo de edad & 0.497 & N.S. \\
\hline Estratos de nivel educativo & $0.092^{*}$ & E.S. \\
\hline $\begin{array}{l}\text { Condición de habla de lengua } \\
\text { indígena }\end{array}$ & $0.085^{*}$ & E.S \\
\hline $\begin{array}{l}\text { Tipo de trabajador } \\
\begin{array}{l}\text { Condición de alta como } \\
\text { trabajador actualmente en el } \\
\text { Lmss }\end{array}\end{array}$ & 0.889 & N.S. \\
\hline
\end{tabular}

Fuente: Elaboración propia a partir de los datos obtenidos

$* 0.05<$ valor $\mathrm{p} \leq 0.1, * * 0.01<$ valor $\mathrm{p} \leq 0.05, * * *$ valor $\mathrm{p} \leq 0.01$;

E.S. = Estadísticamente significativo, N.S. = No es estadísticamente significativo Como puede observarse se presentan diferencias estadísticamente significativas en el nivel de conocimiento de las modalidades de jubilación por tipo de actividad, nivel educativo y condición de habla de lengua indígena. De acuerdo con la estadística asociado con estos grupos, el nivel de conocimiento de las modalidades de jubilación es mayor en lo ganaderos que en los agricultores, a menor edad que a mayor edad y entre quienes no hablan lengua indígena respecto a los que si la hablan, como se muestra en la Tabla 20. 
Tabla 20. Promedio de IMCJ por grupo

\begin{tabular}{|l|l|}
\hline Grupo & Promedio de IMCJ \\
\hline Ganaderos & 13.4 \\
\hline Agricultores & 5.6 \\
\hline Con estudios hasta secundaria & 7.9 \\
\hline Con estudios de bachillerato o mayor & 15.0 \\
\hline Hablantes de lengua indígena & 8.5 \\
\hline No hablantes de lengua indígena & 13.4 \\
\hline
\end{tabular}

Fuente: Elaboración propia a partir de los datos obtenidos

\section{Discusión}

Si bien de acuerdo con el resultado de estudios anteriores, el porcentaje de conocimiento sobre las modalidades de jubilación es muy bajo, se ha estudiado poco acerca de las características de la población a quien están dirigidas estás modalidades; y esto es precisamente lo que el presente trabajo ha aportado en el caso de los trabajadores del campo del municipio de Navojoa. Asimismo, se ha propuesto una medida o índice del grado de conocimiento que está población tiene sobre las distintas modalidades de jubilación de manera conjunta.

Para poder contribuir al conocimiento sobre las modalidades de jubilación es necesario conocer la población a quien irán dirigidas las estrategias de información y comunicación para el logro de este propósito. Se encontró que efectivamente existe bajo nivel de conocimiento en cuanto a las modalidades de jubilación; lo que coincide con lo argumentado por autores como Miranda (2013) y de Ruiz Medina et al (2016), en relación al bajo nivel de conocimiento que existe sobre las modalidades de jubilación, para el caso de México y América Latina.

\section{Conclusión}

Para la realización de este estudio, la muestra de trabajadores del campo se caracteriza por género, edad, nivel de estudios, si habla lengua indígena, actividad que realiza (agrícola o ganadera), condición de derechohabiencia en alguna institución de salud, si se encuentran o 
no dados de alta en el IMSS, si tienen algún plan para la vejez y el grado de conocimiento sobre las Modalidades de jubilación 13, 40 y 43 del IMSS.

En cuanto al género, del total de 120 encuestados $20 \%$ son mujeres y $80 \%$ son hombres, cuya edad promedio es de 57 años. Los rangos de edad son, en las mujeres entre 31 y 90 años y en los hombres entre 26 a 90 años.

El nivel de estudios se encuentra un $81.7 \%$ entre la primaria incompleta hasta el nivel de secundaria.

El $40.8 \%$ de los encuestados habla lengua indígena y sus actividades principales son la agricultura con un $25.8 \%$, siendo el cultivo principal el garbanzo, con $49.8 \%$ y la ganadería con un $74.2 \%$, siendo la especie principal la bovina, con $75.3 \%$.

Actualmente se encuentran dados de alta como trabajador en el IMSS un $18.3 \%$ del total de encuestados, sin embargo, han estado inscritos en el pasado un total de $46.7 \%$, lo cual representa casi la mitad de las personas que pudieran tener acceso a la pensión por cesantía y vejez con esta Institución.

El $41.7 \%$ considera seguir trabajando para hacer frente a la situación de llegar a la vejez, al no contar con un sistema de pensión, mientras que el $20 \%$ considera que algún familiar se hará cargo de ellos al llegar a la vejez y otro $20 \%$ se sostendrá con la ayuda que brinda el gobierno a los adultos mayores.

A partir de la validación del instrumento y de las respuestas a los reactivos en escala ordinal correspondientes, se construyó un índice de conocimiento de las modalidades de jubilación (ICMJ) del IMSS.

El índice de conocimiento se presenta en escala de 0 a 100 para cada trabajador a partir de las sumas de las respuestas a los reactivos en escala ordinal. Dicho índice tiene una media de 11.4 y desviación estándar de 18.4.

Como resultado de la verificación de las hipótesis de investigación, se presentaron diferencias significativas en el nivel de conocimiento de las modalidades de jubilación por tipo de actividad, nivel educativo y condición de habla de lengua indígena. Con respecto al género, grupo de edad, tipo de trabajador (asalariado o no asalariado) y condición de alta actualmente en el IMSS, no se presentan diferencias estadísticamente significativas. 
El nivel de conocimiento de las modalidades de jubilación es mayor en lo ganaderos (10.1) que en los agricultores (3.2), a mayor nivel de estudios y entre quienes no hablan lengua indígena respecto a los que si la hablan.

Estos resultados concuerdan con lo observado por otros autores como Miranda (2013) y de Ruiz Medina et al (2016), acerca el bajo nivel de conocimiento que sobre las modalidades de jubilación se tiene en México y América Latina.

Este trabajo contribuye en la generación de información sobre el conocimiento de las modalidades para la jubilación por cesantía en edad avanzada y vejez, que existen actualmente en el Instituto Mexicano del Seguro Social, sin embargo, el estudio se realiza únicamente en la comunidad de Masiaca, pudiendo aprovechar los resultados de esta experiencia para futuras investigaciones en otras comunidades del estado.

Asimismo, en este estudio se consideraron las actividades de agricultura y ganadería, para los cuales existen modalidades especiales para ellos, pero se pueden realizar otros estudios similares considerando diferentes actividades económicas.

Otra idea es apoyar en la difusión del tema, a través de jóvenes estudiantes universitarios, ya que cada vez son más conscientes de las necesidades de nuestros adultos mayores y más proactivos en cuanto a la búsqueda de soluciones y ayuda a la comunidad.

\section{Referencias bibliográficas}

González, B. G. (1988). Apuntes sobre el futuro de la jubilación en Europa. Documentación laboral, (24). 103-128.

Guzmán, K. (27 de julio de 2020). ¿Cuándo entra en vigor la reforma a la ley de pensiones? Milenio. https://www.milenio.com/negocios/reforma-pensiones2020-entran-vigor-cambios

Instituto Mexicano del Seguro Social. (16 de abril de 2020). Inscripción a la continuación voluntaria al régimen obligatorio en el IMSS. Recuperado de http://www.imss.gob.mx/tramites/imss02007 
Instituto Mexicano del Seguro Social. (s. f.). ¿Tengo derecho a un aguinaldo?

Recuperado de

http://www.imss.gob.mx/pensiones/preguntasfrecuentes/derechoaguinaldo\#: :te $\mathrm{xt}=$ Como\%20pensionado(a)\%20por\%20Cesant\%C3\%ADa,por\%20asignaciones \%20familiares\%20ni\%20ayudas

Instituto Mexicano del Seguro Social. (2018-2019) Prestaciones y Fuentes de Financiamiento de los Regímenes de Aseguramiento del IMSS (Anexo A). Recuperado de http://www.imss.gob.mx/sites/all/statics/pdf/informes/20182019/22$\underline{\text { Anexos.pdf }}$

Instituto Mexicano del Seguro Social. (s.f.) ¿Qué beneficiarios puedo agregar para incrementar mi pensión de Cesantía en Edad Avanzada o Vejez?

Recuperado de http://www.imss.gob.mx/pensiones/preguntas-frecuentes/cuantos beneficiarios-para-incrementar-pension

Instituto Mexicano del Seguro Social. Dirección de Finanzas Coordinación de Estrategia y Planeación Financiera. (2020). Glosario de términos de consulta dinámica (cubos). Autor. Recuperado de http://www.imss.gob.mx/sites/all/statics/pdf/informes/GlosarioCubo.pdf

Instituto Mexicano del Seguro Social. (7 de julio de 2018). Conoce al IMSS. Recuperado de http://www.imss.gob.mx/conoce-al-imss

Instituto Nacional de Estadística y Geografía INEGI. (2015). Número de habitantes. Recuperado http://cuentame.inegi.org.mx/monografias/informacion/son/poblacion/

Lehr, U. (1988). Psicología de la senectud. Proceso y aprendizaje del envejecimiento. Barcelona: Herder, segunda edición.

Miranda, J. (2013). Proyección de pensión personalizada en Chile: Evaluación de su impacto en la decisión de jubilación. Estudios de Economía, vol. 40, no. 2, Recuperado de https://scielo.conicyt.cl/scielo.php?pid=S071852862013000200005\&script=sci_arttextRivera, E. (16 octubre de 2017). Beneficios a familiares de derechohabientes IMSS fallecidos. México. IDC Asesor Fiscal, Jurídico y Laboral. Recuperado de https://idconline.mx/seguridadsocial/2017/10/16/beneficios-a-familiares-dederechohabientes-imss-fallecidos 
Ruiz-Medina, M. I., Borboa - Quintero, M. S. y Cuadras - Borboa, D. M. (2016). Las Pensiones de cesantía-vejez e invalidez de la Ley del IMSS, un análisis teórico práctico en trabajadores de las PYMES. Ra Ximhai, 12 (4), 71-91. Recuperado de https://www.redalyc.org/articulo.oa?id=46146927005

Sáez, N. N., Meléndez, M. J., Aleixandre, R.M. y De Vicente M. P. (1990). Preparación para la Jubilación. Recuperado de https://www.uv.es/melendez/envejecimiento/Preparacionalajubilacion.pdf

Sánchez-Castañeda, A. y Morales, R. M. (2018). Pensión o Jubilación. Derechos de las personas pensionadas y jubiladas. Nuestros derechos, (pp1-3). Recuperado de https://archivos.juridicas.unam.mx/www/bjv/libros/11/5486/5.pdf

Serna-Cantú, V., Alcántar-Román, T. y Gaytán-Duarte, E. (2016). Desarrollo de la Ciudad de Navojoa Sonora. Vinculatégica EFAN, 2(1), 1661-1679. Recuperado de

http://www.web.facpya.uanl.mx/vinculategica/Revistas/R2/1815\%20\%201833\%20\%20Desarrollo\%20de\%20la\%20ciudad\%20de\%20Navojoa\%20S onora.pdf

Sonora apuntes para la historia de la educación. (2016, septiembre). Sonora apuntes para la historia de la educación. Recuperado de: https://www.unilider.edu.mx/sonora-apuntes-para-la-historia-de-la-educacion-tomoii/

Tipos de jubilación que se pueden solicitar. (2016, 5 julio). México. Proyecta Promociones. Recuperado de https://www.proyectapensiones.com.mx/post/tipos-dejubilaci\%C3\%B3n-quese-pueden-solicitar

\section{Como citar:}

Rivas Vega, M. ., González Barajas, M., \& Clark Coronado, P. (2021). Jubilaciones IMSS por cesantía en edad avanzada y vejez, sector agropecuario, Municipio de Navojoa, Sonora. Revista De Investigación Académica Sin Frontera: División De Ciencias Económicas Y Sociales, (36). https://doi.org/10.46589/rdiasf.vi36.399 
D Dialnet

latinde

catsoger

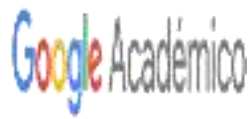

DORA
CiteFactor $+3$
REDIB

1. IJIFACTOR

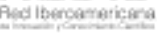

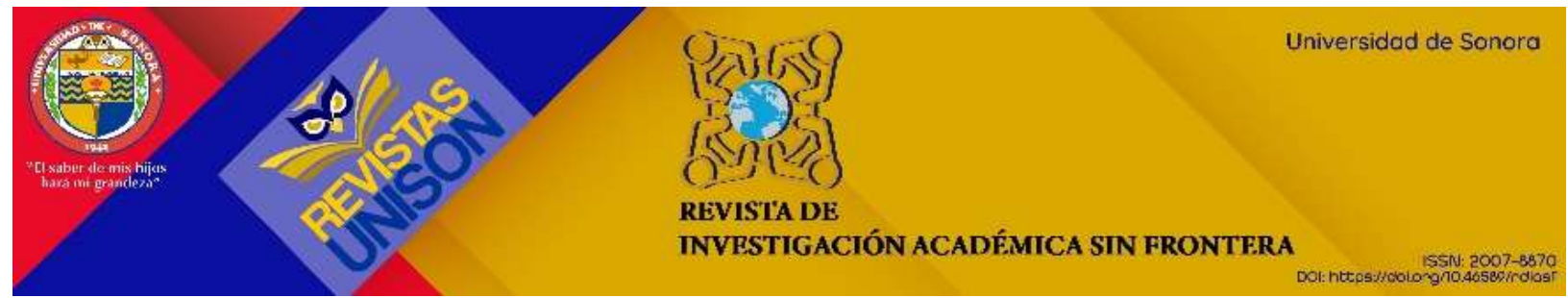

